\title{
Ebola: How well is the UK prepared?
}

Rebecca Coombes and Sophie Arie sum up the latest information about the risk of Ebola arriving in the UK and how health services are being prepared to manage suspected cases

\section{Rebecca Coombes magazine editor, Sophie Arie freelance journalist}

The BMJ, London, UK

\section{What is the scale of the public health threat that the UK is preparing for?}

The government expects a handful of cases of Ebola virus disease in the UK over the next three months and it hopes, through screening at airports ${ }^{1}$ and other points of entry, to identify passengers coming from the affected region (there are no direct flights and fewer than 1000 arrived via flights from other European capitals in September) who could develop the disease once in the UK, even if they have no symptoms on arrival. Any passengers Public Health England (PHE) considers to be at an increased risk of infection will be given information and advised not to go to a hospital or GP if they develop symptoms but to call 111 or 999 . Posters are also being placed at airports to encourage travellers who may have travelled indirectly to the UK to refer themselves to PHE for screening. ${ }^{2}$

People who are considered to be at a high risk, because they have been working with infected people or have had contact with bodies or soiled equipment, will be asked to call PHE directly so that they do not pass through NHS services unnecessarily. ${ }^{3}$ The government says that this system should mean that UK public health officials can identify $89 \%$ of all those arriving on the main routes to the UK. Jeremy Hunt, the health secretary, acknowledged in a briefing to parliament on 14 October that "no screening procedure will be able to identify $100 \%$ of people arriving from Ebola affected countries." PHE has also issued guidance for universities - where students, including many from west Africa, have just arrived for the new academic year — on being vigilant and taking precautions, including informing and equipping staff who clean student accommodation to be on the alert for any signs that a student may be unwell. ${ }^{4}$

\section{What are the chances of someone infected with Ebola virus presenting unexpectedly at a general practice, hospital, or elsewhere?}

Extremely low-firstly, because only a few cases are expected to be imported to the UK and, secondly, because if the government's screening process works, those who are aware they are at risk should know who to call for advice and would be brought by ambulance to an acute care service that would be notified in advance.

General practices, hospitals, schools, and universities are much more likely to find themselves dealing with false alarms. "Most of the cases that present with relevant symptoms will not turn out to be Ebola," says David Mabey, professor of communicable diseases at the London School of Hygiene and Tropical Medicine. Mabey emphasises the need for testing suspected cases to be made as fast as possible "otherwise there could be a huge use of resources to isolate people who do not have Ebola."

A few false alarms have been reported in recent days. A man who had recently travelled to the UK from Sierra Leone presented at the emergency department of University Hospital Lewisham, in south east London, and was treated as a suspected case of Ebola infection until test results were negative. And a man from Nigeria who presented with a fever at University Hospitals Coventry and Warwickshire was found to have another infectious disease.

One fact that will rule out many suspected cases is that if someone has caught Ebola virus they will have symptoms within 21 days. Any symptoms they develop after that period will not be due to Ebola virus. Another fact that helps control Ebola is that patients are not infectious until they develop symptoms. ${ }^{5}$ Patients become more infectious as their disease progresses; the risk of transmission through casual contact with a mildly ill patient is low. However, high concentrations of virus may occur in diarrhoea and vomit, and the clinical environment can become highly contaminated. ${ }^{6}$

\section{Are health services in the UK prepared for suspected Ebola cases?}

"They should be," says David Heymann, chair of PHE and professor of infectious disease epidemiology at London School of Hygiene and Tropical Medicine. All acute health services have been given detailed instructions on what to do and how to protect themselves. "Staff here are all on the alert, but that is no guarantee they will all follow the guidelines," adds Heymann. The two nurses who have become infected in the US after treating a man with Ebola show that mistakes can be made even 
in the countries that are best prepared and equipped to care for patients. Yet, as Mabey points out, those who follow the guidance to the letter, at every stage, will be safe, even if working conditions are less than ideal. "Oliver Johnson, a young English doctor, has been running a treatment centre in Freetown [the Liberian capital] for six months and not been infected," Mabey says.

Most of the guidance for health workers has been in place for a long time because there has always been a low risk of Ebola or other viral haemorrhagic fevers being imported to the UK. PHE is constantly updating its risk assessment algorithm to help health professionals assess, rapidly diagnose, and safely manage a patient with suspected Ebola infection. ${ }^{7}$ It confirmed that its imported fever service is now receiving 50-80 calls a week from clinicians seeking guidance when treating a patient with fever symptoms. In July the average was around 15 calls.

Lessons learnt from two mock Ebola emergencies on 12 and 13 October are being circulated to all emergency departments and acute care services. Similarly, ambulance services nationwide are practising their response procedures.

\section{What is the risk for health workers who come into contact with a patient with Ebola}

Acute trusts do not need to stock up on full body suits with breathing apparatus similar to those used by Médecins Sans Frontières in west Africa.

Guidance from the Advisory Committee on Dangerous Pathogens sets out protection standards for dealing with suspected cases of Ebola in the UK, which include hand hygiene, double gloves, plastic apron, fluid repellent surgical facemask, and eye protection.

"What's important is to prevent any infected fluids getting into the eyes or lips," says Mabey. "We don't think it goes through intact skin."

It's a question of taking sensible precautions, he explains. These are using simple protective clothing (a mask, goggles, gloves, and a gown) and knowing how to remove and dispose of it safely because even small quantities of infected body fluids can transmit the disease. Good training is essential. Correct protective equipment and meticulous attention to guidance on protection procedures at every stage can ensure health workers remain safe.

Preparation is key. Every acute hospital should have a local plan covering everything from identifying and preparing a quarantine area so it is ready for use and making sure staff know where it is, through considering the need for interpreters, to the disposal of contaminated bodily fluid. There should be an education programme for staff and regular drills, including for reception of patients at risk and donning and safely removing personal protective equipment. One senior doctor with experience of treating patients with viral haemorrhagic fever told The BMJ: "Theoretically every acute trust should have appropriate equipment, boots, scrubs, theatre gowns, masks. But you need to think about where this kit needs to be if someone walks in vomiting with suspected Ebola. You don't want to be doing this when the patient is sitting opposite you in the room."

\section{What is the latest guidance for primary care workers?}

General practices, out of hours centres, and walk-in centres are unlikely to see imported cases of Ebola from west Africa, but staff are advised to remain vigilant.

A patient who telephones-Anyone who has visited an affected country in the past 21 days and reports a fever of more than $38^{\circ} \mathrm{C}$ within the past 24 hours should not visit health premises. Instead a clinician should refer the patient to the local acute hospital.

A patient visits any primary care service-Any person unwell and newly returned from an area affected by Ebola within the past 21 days should not sit in the general waiting area and should be isolated in a side room (cleared of removable items to reduce decontamination requirements if Ebola is confirmed). Urgent clinical advice needs to be sought from a local microbiologist, virologist, or infectious disease physician. Staff in contact with the patient should be wearing appropriate protection-gloves, plastic apron, surgical facemask, and visor.

Patients should be sent to hospital in an ambulance, and paramedics should be warned of the possibility that the patient is infected with Ebola virus. GPs also need to alert the hospital that Ebola is suspected.

GPs must alert the local health protection team for advice, including decontamination procedures. It may be necessary to quarantine the room for up to 24 hours while Ebola is confirmed or excluded. No one should use the room or touch any potentially contaminated areas, including toilets and door handles, until they are decontaminated. Public areas where a patient has just passed through do not need to be specially cleaned.

If Ebola is confirmed by laboratory testing, the local health protection team will review any contacts at the primary care service.

\section{What should secondary care staff do?}

The College of Emergency Medicine issued joint guidance with PHE on 15 October for emergency department staff. ${ }^{6}$ It recommends a simple checklist from the Centers for Disease Control and Prevention in the US. ${ }^{8}$ The list breaks down into three areas: detect, protect, and respond.

Patients arriving by ambulance-The hospital should be alerted by ambulance crew before the patient arrives, giving time for staff to identify a side room and access appropriate protective equipment. This should be worn by staff meeting the ambulance.

Patient presenting at an emergency department-Staff must adapt a "talk but don't touch" approach to the patient. Rapid identification and isolation of patients with suspected Ebola infection are essential. Suspected patients should be asked two initial screening questions: have you travelled from one of the affected areas or cared for someone with Ebola within the past 21 days and do you have a fever $\left(>38^{\circ} \mathrm{C}\right)$ or have had a fever in the past 24 hours? Patients who answer yes should be isolated in a single room and have a full history taken by a clinician wearing appropriate protective clothing and trained in its use. What if the patient has been sitting in the public area?-The patient should be isolated as above, and the area in a waiting room cordoned off until the patient is assessed. If the patient is considered likely to be infected with Ebola virus, staff should get guidance from the hospital lead for infection control and notify the local PHE health protection team. Trained staff should decontaminate the area. The names of patients and staff who 
might have come into physical contact with the patient or body fluids should be documented.

Once a patient with suspected Ebola has been isolated-The infection consultant will discuss testing with the imported fever service. The advice is not to wait for Ebola tests results before doing other relevant diagnostic tests, such as for malaria.

\section{Diagnostics}

If someone is thought to be highly likely to have Ebola, a result can be given within four hours of the specimen being received by the laboratory. Testing is done by the imported fever service, a 24 hour, seven day a week service, based at the Rare and Imported Pathogens Laboratory in Porton Down, Wiltshire. The service has tested 10-15 suspected cases of viral haemorrhagic fever every week for the past three months. PHE says it has sufficient capacity to manage this increase in testing volume. For advice on testing possible cases of Ebola virus disease, clinicians need to contact their local infectious disease physician or consultant microbiologist or virologist, who will assess urgency and advise on the correct samples to take (the minimum samples are serum and EDTA blood, and ideally a urine sample) and how to transport the samples.

\section{What happens if a patient tests positive for Ebola virus?}

The key teams alerted are the outbreak control teams and duty doctor at PHE Colindale, senior government officials, and the clinical team at the high level isolation unit, Royal Free Hospital, the UK's specialist centre for treating the most dangerous infectious diseases. In Scotland, there are well rehearsed protocols for the isolation, transport, investigation, and medical care of any patients with suspected Ebola infection. There are four infectious disease units in Scotland providing round the clock specialist consultant cover, where patients with Ebola can be cared for until they are transferred to the Royal Free.

Early detection and good care much improve a patient's chance of recovery. Symptoms start with a fever, aches, and headache and then develop into diarrhoea, vomiting, uncontrolled internal bleeding, bleeding from the eyes, nose, and ears, and organ failure. Intravenous fluids, in large quantities, can help the patient fight the virus. Blood transfusions may also be necessary. Dialysis for renal failure is considered high risk for health workers and the Royal Free Hospital has therefore ruled out offering this.

Although there is no approved cure or treatment for the virus, the one patient treated so far in the UK was given an experimental treatment, and PHE says that "In some instances, clinicians treating individuals with Ebola may source and decide to use an experimental drug, such as Zmapp." ${ }^{\text {L }}$ Laboratory testing of Zmapp has shown that it is most effective if given to a patient within days of being infected and much less effective if symptoms are advanced.

\section{Could the Ebola threat to the UK increase?}

The chief medical officer has said that although the threat is currently low, the government should make preparations for a possible increase in the risk level. There are plans in place to expand capacity for patients with Ebola in Newcastle, Liverpool, and Sheffield, making a total of 26 beds available in the UK if needed. Such a surge in cases could come from a large increase in the numbers of cases reported in west Africa, an increase in the numbers of countries where cases are reported and outbreaks are not well controlled, or a breakdown of security in the countries at the heart of the epidemic.

Ebola is not airborne and, unlike other new infectious diseases that have emerged in recent years, experts are not concerned that this virus could mutate and become airborne and therefore far more easily spread and much harder to control. The Ebola virus is still the same as it was in 1976. Because the current outbreak is extraordinary, nothing can be ruled out. The disease has infected many more people than it ever has previously and it is possible, says Mabey, that as a result of passing through more humans a particularly infectious strain could emerge.

Competing interests: We have read and understood BMJ policy on declaration of interests and have no relevant interests to declare Provenance and peer review: Commissioned; not externally peer reviewed.

Mabey D, Flasche S, Edmunds WJ. Airport screening for Ebola. BMJ 2014;349:g6202 .

2 Public Health England. Returning from west Africa? Information about Ebola. www.gov. uk/government/uploads/system/uploads/attachment_data/file/363681/Ebola_A3 poster. pdf

3 Public Health England. Enhanced Ebola screening process. www.gov.uk/government/ news/public-health-england-enhanced-ebola-screening-process.

4 Public Health England. Ebola: advice and risk assessment for universities and further education establishments. 2014. www.gov.uk/government/uploads/system/uploads/ attachment data/file/355421/Ebola advice for further educational establishments 20140911.pdf.

5 Public Health England. Ebola Q\&A, October 2014. www.gov.uk/government/uploads/ system/uploads/attachment_data/file/363883/14_10_14_Ebola_QA_For_Public.pdf. system/uploads/attachment_data/file/363883/14_10_14_Ebola_QA_For_Public.pdf.
College of Emergency Medicine, Public Health England. Ebola guidance for emergency departments. CEM, 2014.

7 Public Health England. Viral haemorrhagic fevers risk assessment. 2014. www.gov.uk/ government/uploads/system/uploads/attachment_data/file/354641/VHF_algorithm_10_ 09_2014.pdf].

8 CDC. Detailed hospital checklist for Ebola preparedness. www.cdc.gov/vht/ebola/pdf/ hospital-checklist-ebola-preparedness.pdf.

Cite this as: BMJ 2014;349:96288

(c) BMJ Publishing Group Ltd 2014 